\title{
Induced changes in solvent structure by phospholipid monolayer formation at a liquid-liquid interface
}

\author{
R.A. Walker ${ }^{1}$, D.E. Gragson ${ }^{2}$, G.L. Richmond * \\ Department of Chemistry, University of Oregon, Eugene OR 97403, USA
}

\begin{abstract}
Vibrational sum frequency spectroscopy has been used in conjunction with dynamic surface tension measurements to study formation of a 1,2-dilauroyl-sn-phosphatidylcholine (DLPC) monolayer at a water-carbon tetrachloride interface. Surface tension measurements show that an aqueous solution of liquid crystalline phosphocholine vesicles (4.5 $\mu \mathrm{M}$ DLPC) requires several hours to form a tightly packed, fully equilibrated monolayer of DLPC monomers. Vibrational spectra of the interfacial region at different stages in the monolayer formation process indicate that the solvent structure undergoes dramatic re-organization as the monolayer forms. Initial adsorption of DLPC monomers severely disrupts the interfacial hydrogen bonding. Intensity in the $\mathrm{OH}$ stretching region oscillates in a systematic fashion during the first $2 \mathrm{~h}$ of monolayer formation before finally settling to a level characteristic of the fully equilibrated monolayer. Frequency shifts of the $\mathrm{OH}$ stretching vibration show that water molecules with their $\mathrm{C}_{2}$ axes aligned parallel to the interface experience a markedly different environment than those water molecules aligned perpendicular to the interface. This difference is attributed to the effect of the adsorbed, zwitterionic DLPC head-groups which, if aligned parallel to the interface, can stabilize in-plane water molecules.
\end{abstract}

\section{Introduction}

Surfactant monolayers at liquid surfaces play a central role in many processes including lubrication, emulsification, and detergent action [1-3]. The timescale over which these monolayers form can be quite long. Monolayers formed from ad- sorption of surfactants from an underlying bulk solution can require minutes, hours or even days to achieve equilibrium [4]. To study the kinetics of monolayer formation a number of different techniques have evolved, most of which measure the time-dependent surface tension as a function of temperature, bulk surfactant concentration and ionic strength $[5,6]$. While these methods provide valuable information about the rate at which a monolayer forms, they remain thermodynamic in nature, thus unable to shed insight into changes in molecular structure that accompany monolayer 
formation. In contrast, second order non-linear optical spectroscopy can directly probe how the interfacial molecular environment evolves during the monolayer formation process [7]. In this report we employ vibrational sum frequency spectroscopy (VSFS) to examine the changes in interfacial solvent structure induced by formation of a phospholipid monolayer at a liquidliquid interface.

Use of second order non-linear optical spectroscopy to study surfaces (i.e. VSFS, second harmonic generation ( $\mathrm{SHG})$ ) has blossomed in recent years due to the interfacial specificity of these techniques [8-12]. Surprisingly, little work has focused on the time-dependent structural behavior of interfacial processes. Shen and coworkers reported using second harmonic generation to follow the adsorption of sodium dodecyl napthalene sulfonate (SDNS) to a freshly cleaned air-water interface [7]. They found that the SDNS optical data tracked dynamic surface tension data quite closely and concluded that SHG represented an optical, in situ means with which to monitor adsorption kinetics and to determine final monolayer composition. SHG data also allowed the authors to determine the gross orientation of the adsorbed chromophore. This study marked the first application of interfacially specific, non-linear optical spectroscopy to issues of dynamics at a liquid surface. However, the data also highlighted a major drawback associated with SHG: although this technique can disclose the orientation and surface concentration of adsorbed chromophores, it lacks the ability to provide information about detailed conformation and orientation of interfacial solvent molecules. Acquiring this type of data requires not only surface specificity but also the molecular specificity that is found in VSFS spectra [11]. Like SHG, VSFS is a second order, non-linear optical technique that samples interfaces between isotropic media. However, spectra acquired by VSFS contain molecularly specific information because they reveal the vibrational structure of all species in the interfacial region.

This report contains the first detailed look at the time-dependent changes in molecular solvent structure as a phospholipid monolayer forms at a liquid-liquid interface. Monitoring the vibrational spectra of the interfacial water molecules as phospholipid monomers adsorb to aqueous$\mathrm{CCl}_{4}$ interface uncovers complex changes in the interfacial solvent structure which can not be inferred from dynamic surface tension data. We find that initial adsorption of phospholipid monomers disrupts the hydrogen bonding network of interfacial water molecules, and we observe distinct differences between water structure aligned in the plane of the interface and water structure normal to the interface. A large red shift in the vibrational spectra of in-plane water molecules suggests that the zwitterionic phospholipid head-groups set up small, microscopic potential gradients parallel to the interface which strengthen the hydrogen bonding in this dimension.

\section{Experimental}

The experiments described in this work examine the changes in water structure at an aqueous-carbon tetrachloride interface during formation of a phospholipid monolayer. The phospholipid used in these studies, 1,2-dilauroyl$s n$-phosphatidylcholine (DLPC, Fig. 1), belongs to a family of saturated, symmetric diacyl phosphocholines. In aqueous solution these molecules spontaneously aggregate in bilayer structures which, upon sonication above the bilayer gelliquid crystalline transition temperature, rearrange to form closed shell, bilayer vesicles [13]. At interfaces these vesicles break apart to form monolayers of phosphocholine monomers [14-

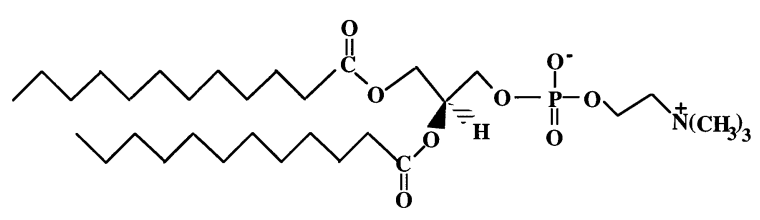

Fig. 1. A picture of 1,2-dilauroyl-sn-glycero-phosphocholine (DLPC), the phosphocholine used in these studies. DLPC has a zwitterionic head-group at neutral $\mathrm{pH}$ and a pair of saturated, symmetric, $\mathrm{C}_{12}$ acyl chains. 
17]. The rate at which these monolayers form depends quite sensitively on the phase of the vesicle bilayer [16,18]. Vesicles in their gel (or frozen) state form monolayers very slowly, requiring days to establish equilibrium. Once above their transition temperature, however, vesicle bilayers exist in a more fluid-like, liquid crystalline state. Monomers dissociate from vesicles much more quickly, adsorbing to the interface to form equilibrated monolayers in a matter of hours. With a transition temperature of $-1{ }^{\circ} \mathrm{C}$, DLPC vesicles used in these room temperature experiments were in their fluid-like, liquid crystalline state. At aqueous phosphocholine concentrations of $4.5 \mu \mathrm{M}$, equilibrated monolayers formed in $\sim 6 \mathrm{~h}$.

Stock solutions of DLPC vesicles were prepared by suspending a given amount of the phospholipid in water buffered to a $\mathrm{pH}$ of 7.0 with sodium phosphate $(\sim 10 \mathrm{mM}$ sodium phosphate). The aqueous solutions were then sonicated at room temperature $\left(23^{\circ} \mathrm{C}\right)$ until the solutions became clear. Dynamic light scattering experiments showed the DLPC vesicles to be $100-150 \mathrm{~nm}$ in diameter, indicating that they were probably multi-lamellar in nature. Typical stock solution concentrations ranged from 0.5 to $1.0 \mathrm{mM}$ in phosphocholine concentration.

Dynamic surface tension measurements were carried out with a Pt Wilhelmy plate-microbalance assembly [6]. Experiments began by monitoring the interfacial tension of the neat aqueous $-\mathrm{CCl}_{4}$ interface. The aqueous phase consisted of nanopure water saturated with $\mathrm{CCl}_{4}$ and buffered to $\mathrm{pH} 7.0$ with a sodium phosphate solution. After establishing interfacial stability and purity, we made a small volume addition of the DLPC vesicle stock solution to the aqueous phase bringing the aqueous DLPC concentration up to the desired value. Additions usually required $\sim 100 \mu \mathrm{l}$ dispersed in uniform drops upon the water surface. As the monolayer formed the surface tension dropped and after several hours, the surface tension begins to approach an asymptotic limit.

To probe molecular structure at the interface as the monolayer formed, we employed vibrational sum frequency spectroscopy in a total in- ternal reflection (TIR) geometry [19]. Given its molecular and interfacial specificity, VSFS has developed into a powerful technique for probing solid-liquid [8], liquid-air [10] and liquid-liquid interfaces [19]. The method involves two coherent optical fields - typically one fixed frequency visible and one tunable infrared-converging spatially and temporally at the interface. When the infrared radiation is resonant with an allowed vibrational transition of a molecule at the interface, the two waves interact through the resonant term of the second order non-linear susceptibility $\left(\chi^{2}\right)$ to create a third optical field equal in energy to the sum of the visible and infrared energies.

A TIR geometry enhances sensitivity by up to three orders of magnitude due to the creation of an evanescent wave at the interface [20,21]. In a TIR geometry, the visible and infrared beams pass through the high index medium $\left(\mathrm{CCl}_{4}\right)$ and the sum frequency (SF) signal is collected in reflection. Spectra presented in this paper come from one of two polarization conditions. Detecting the S-polarized SF signal arising from S-polarized visible and P-polarized infrared $\left(\mathrm{S}_{\text {sum }} \mathrm{S}_{\mathrm{vis}} \mathrm{P}_{\text {ir }}\right.$ or $\left.\mathrm{SSP}\right)$ probes vibrational motion having a component normal to the plane of the interface. A spectrum recorded under $\mathrm{S}_{\text {sum }} \mathrm{P}_{\mathrm{vis}} \mathrm{S}_{\text {ir }}$ (SPS) conditions samples vibrational motion in the plane of the interface.

The laser system used to acquire the data presented in this report has been described previously [22]. Briefly, a fraction of the $800 \mathrm{~nm}$ output from a regeneratively amplified Ti:Sapphire laser (Coherent, Quantronix) pumped a white light generation-optical parametric amplifier assembly to provide a source of tunable infrared radiation $\left(3-6 \mu \mathrm{J}, 18 \mathrm{~cm}^{-1}\right.$ FWHM). The remaining $800 \mathrm{~nm}$ light was used as the fixed frequency visible field. An important aspect of this system was its $1 \mathrm{kHz}$ repetition rate. By averaging only 50 shots per data point, a spectrum from 2800 to $3650 \mathrm{~cm}^{-1}$ can be recorded every $3 \mathrm{~min}$. Given that a DLPC monolayer requires hours to form, this time resolution is sufficient to follow the evolution of interfacial solvent structure during DLPC monolayer formation. 


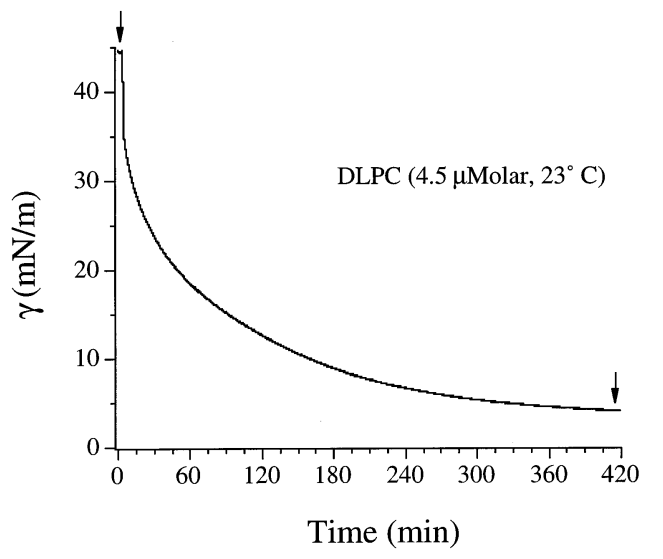

Fig. 2. Dynamic surface tension measurement tracking the formation of a DLPC monolayer at the water-carbon tetrachloride interface. Bulk DLPC aqueous concentration is 4.5 $\mu \mathrm{M}$ and the temperature is $23^{\circ} \mathrm{C}$. After a steep initial descent during the first hour of monolayer formation, the surface tension begins to decay in an exponential fashion, slowly approaching the asymptotic limit characteristic of a tightly packed monolayer.

\section{Results and discussion}

\subsection{Dynamic surface tension measurements}

A representative dynamic surface tension measurement appears in Fig. 2. In this experiment, addition of stock solution raised the bulk DLPC concentration to $4.5 \mu \mathrm{M}$. The surface tension dropped steeply over the first $30 \mathrm{~min}$ and after 60 min assumed an exponential decay, slowly approaching an equilibrium value of $\sim 4 \mathrm{mN} / \mathrm{m}$. The experiment shown in Fig. 2 ended with a surface pressure of $41 \mathrm{mN} / \mathrm{m}$ which is characteristic of monolayers having surface concentrations of $1.8 \times 10^{14}$ molecules $/ \mathrm{cm}^{2}$, or, equivalently, 55 $\AA^{2} /$ molecule [23] (surface pressure reflects the difference between the surface tension of the neat interface $\left(\gamma_{0}=44.5 \mathrm{mN} / \mathrm{m}\right)$ and the terminal surface tension of the interface after monolayer formation). This terminal surface concentration agreed with results from surface pressure measurements of phosphocholine monolayers at other organic-aqueous interfaces [5,24,25].

Several important issues are evident in Fig. 2. First, the data show that the equilibrated DLPC monolayer requires several hours to form from a solution of liquid crystalline DLPC vesicles. This behavior is quite general for liquid crystalline vesicles composed of saturated, symmetric diacyl phosphocholines [14,23]. For aqueous solutions containing gel state vesicles, monolayer formation can require days and the equilibrated monolayers are considerably expanded [23]. Both the gel and liquid crystalline situations stand in sharp contrast to formation of a monolayer from a solution of simple, soluble surfactants (e.g. sodium dodecyl sulfate, $\mathrm{SDS}$ ): starting from a neat aqueous $-\mathrm{CCl}_{4}$ interface, a small volume addition of SDS stock solution leads to formation of a complete monolayer in approximately $30 \mathrm{~min}$ [19].

Second, during the formation of a DLPC monolayer, the surface tension decays smoothly over time. Contained in the data is valuable information about the rate at which the monolayer forms, and we have shown how the kinetics of monolayer formation may be described by a simple first order kinetic model [18]. Absent in this macroscopic analysis, however, are the details about how the molecular structure of the monolayer and the surrounding solvent evolves during monolayer formation. Experiments discussed in this paper provide evidence of complex changes in interfacial solvent environment which can not be inferred from the dynamic surface tension data.

\subsection{Molecular structure of the neat interface and full monolayer}

A dynamic VSFS experiment consists of (1) establishing a neat aqueous- $\mathrm{CCl}_{4}$ interface and recording a VSF spectrum to establish interfacial purity; (2) adding a small volume of DLPC stock solution to the aqueous phase and (3) a spectrum is recorded every few minutes. Shown in Fig. 3 are two spectra taken at the times marked on the dynamic surface tension experiment depicted in Fig. 2. Appearing in the upper panel of Fig. 3 is an SSP spectrum of the neat aqueous $-\mathrm{CCl}_{4}$ interface. The bottom panel (Fig. 3b) depicts an SSP spectrum of an equilibrated, tightly packed DLPC monolayer.

In the SSP spectrum of the neat interface, more than $90 \%$ of the spectrum's intensity lies under the $\mathrm{OH}$ stretching feature centered at $3180 \mathrm{~cm}^{-1}$. 
Interfacial tension measurements indicate that the boundary between the two solvents is free of contaminants, although we still observe weak intensity in the $\mathrm{CH}$ stretching region $(\sim 2970$ $\mathrm{cm}^{-1}$ ) implying the presence of trace, surface active impurities. Despite rigorous cleaning procedures and perfect wetting behavior on the quartz cell, this feature invariably appears in the $\mathrm{CH}$ stretching region of the nominally neat interface. The concentration of this impurity lies below the detectable limits of various diagnostic techniques (FTIR, NMR, UV-VIS) used to assess the purity of the solvents employed in these experiments. Assuming these limits to lie in the nm (or ppb) range and taking into account inaccuracy of measuring the tension of the neat interface $( \pm 0.5$ $\mathrm{mN} / \mathrm{m}$ ), we estimate a contaminant surface concentration of $\sim 1 \times 10^{11}$ molecules $/ \mathrm{cm}^{2}$ or $\sim$ 0.001 monolayers. We conclude that such low surface concentrations of trace contaminants will not significantly affect our measurements. That such impurities show up at all reflects the extreme sensitivity of the VSFS-TIR experiments carried out with the optical system described above.

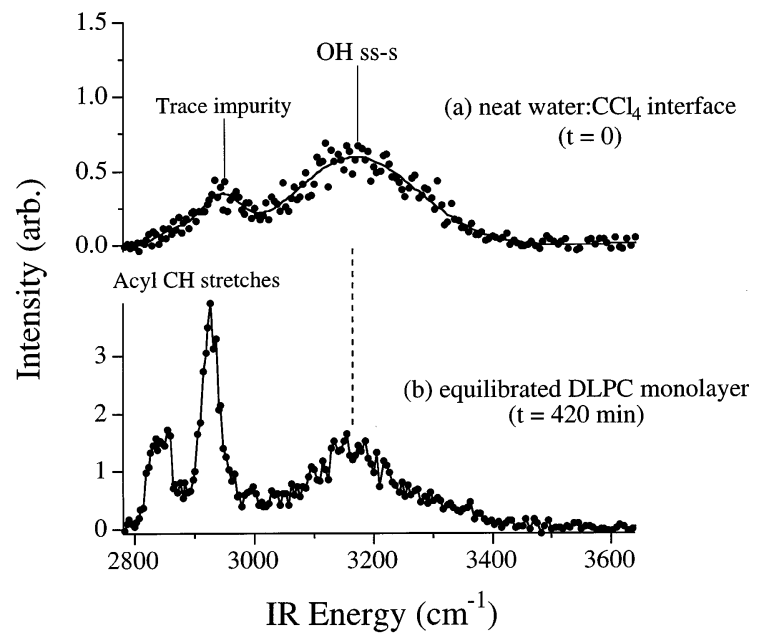

Fig. 3. VSFS spectra of (a) the neat aqueous-carbon tetrachloride interface and (b) the aqueous-carbon tetrachloride interface to which a tightly packed DLPC monolayer has been adsorbed. Spectra were recorded under SSP conditions and intensities have been corrected for power. Spectra were fit using Lorentzian profiles and assignments were based on previous VSFS, IR, and Raman studies (see text).

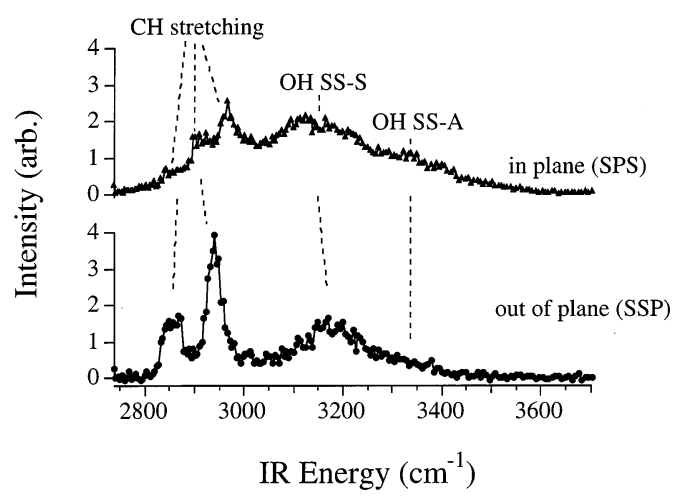

Fig. 4. VSFS spectra of an equilibrated DLPC monolayer recorded under (a) SPS and (b) SSP polarization conditions. The choice of SPS samples vibrational motion in the plane of the interface while SSP samples vibrational motion perpendicular to-or out-of-plane of - the interface. Spectra have been corrected for power and differences in Fresnel coefficients between the two polarization conditions.

This report considers only how the water structure evolves during DLPC monolayer formation at the aqueous $-\mathrm{CCl}_{4}$ interface. Spectra of the equilibrated DLPC monolayer taken under different polarization conditions are shown in Fig. 4. These spectra have been corrected for differences in incident IR power and for differences in their respective Fresnel factors. Intensity in the water region of the SPS spectrum (lower panel, Fig. 4) extends to higher frequency than it does in the SSP spectrum. These two polarization choices sample vibrational motion-parallel (SPS) and perpendicular (SSP) - to the interface, and differences in the spectra imply an anisotropy in the interfacial solvent environment. To interpret the spectroscopic changes in water vibrational structure which accompany DLPC adsorption to the interface, we briefly summarize the results of infrared, Raman and VSFS studies of water structure at interfaces.

The topic of water structure at interfaces has received considerable attention in recent years [26-30]. VSF spectra of water at interfaces show up to three distinct features. Assignment of these VSF bands was based on the findings of earlier Raman and infrared studies of bulk water [3133]. A broad band at $3200 \mathrm{~cm}^{-1}\left(>200 \mathrm{~cm}^{-1}\right.$ FWHM) has been assigned to the in-phase sym- 
metric stretching modes of coupled water molecules in a symmetric, hydrogen-bonded environment (OH SS-S) [31]. This feature indicates a high degree of hydrogen bond ordering such as that found in bulk ice [32]. The OH SS-S band appears in VSF spectra of water at both air-water and water-organic interfaces [27,30].

A second broad band ( $\mathrm{OH}$ SS-A) sometimes appears near $3400 \mathrm{~cm}^{-1}$ ( $200 \mathrm{~cm}^{-1}$ FWHM) in the VSF spectra of aqueous surfaces. The exact assignment of this feature is uncertain. Some researchers attribute the band to the symmetric stretch of coupled water molecules in an asymmetric hydrogen-bonded environment [31] while others claim that the feature arises from $\mathrm{OH}$ stretches of water molecules having bifurcated hydrogen bonds [33]. Regardless, spectral intensity in the $3400 \mathrm{~cm}^{-1}$ region implies greater disorder in the intermolecular hydrogen bonding and a correspondingly more 'liquid-like' environment. The spectra in Fig. 4 suggest that in the presence of a fully formed DLPC monolayer, water structure is more disordered parallel to the interface compared with the perpendicular dimension as evidenced by noticeable intensity above 3300 $\mathrm{cm}^{-1}$ in the SPS spectrum. All efforts to fit the SSP spectrum in Fig. 4 to two features show that a second feature in the $\sim 3300-3400 \mathrm{~cm}^{-1}$ region would contribute less than $5 \%$ to the observed $\mathrm{OH}$ intensity, consistent with earlier VSFS studies [34].

The third feature in VSF spectra of water at interfaces sometimes appears at $3680 \mathrm{~cm}^{-1}$ and is much sharper $\left(60 \mathrm{~cm}^{-1} \mathrm{FWHM}\right)$ than the $\mathrm{OH}$ SS-S and SS-A bands [30]. This narrow peak results from 'free' or 'dangling' $\mathrm{OH}$ groups at an interface and implies the existence of water molecules that are missing one of their hydrogen bonds. This band also appears in spectra of the neat aqueous- $\mathrm{CCl}_{4}$ interface although it disappears in the presence of a charged surfactant [35]. Previous studies have also shown that any amphiphile - charged or uncharged - alters the surrounding solvent structure at air-water and liquid-solid interfaces such that the free $\mathrm{OH}$ band disappears from VSF spectra.[29,30]

VSFS spectra of the water- $\mathrm{CCl}_{4}$ interface at different stages of DLPC monolayer formation generally show the OH SS-S to be the dominant feature in the water stretching region. Exceptions to this generalization only occur in SPS spectra acquired in the first few minutes of monolayer formation. In these early SPS (in-plane) spectra, the OH SS-A can carry up to $50 \%$ of the observed $\mathrm{OH}$ intensity in SPS spectra. Generally, however, the OH SS-A band contains no more than $20 \%$ of the observed integrated $\mathrm{OH}$ signal in SPS spectra after the initial stages of monolayer formation. In SSP (out-of-plane) spectra uncertainty in fitting a feature as broad as the OH SS-S band precludes us from saying that the OH SS-A band is absent, but we can place an upper limit of $5 \%$ to any contribution this feature may make to the observed intensity. The $3680 \mathrm{~cm}^{-1}$ free $\mathrm{OH}$ feature was not present during the DLPC adsorption process and will not be considered further. The analysis which follows will focus exclusively on the intensity and frequency fluctuations in the $\mathrm{OH}$ SS-S band which accompany formation of the DLPC monolayer. Further studies are underway to investigate the origin of the observed differences in in-plane vs. out-of-plane solvent anisotropy.

\subsection{Temporal dependence of $\mathrm{OH} S \mathrm{~S}-\mathrm{S}$ intensity}

An experiment begins with the addition of a small volume of DLPC stock solution to initiate monolayer formation. Radical changes in the interfacial water environment immediately ensue. Fig. 5 depicts the time dependence of the $\mathrm{OH}$ SS-S in both the in-plane (Fig. 5a) and out-ofplane (Fig. 5b) dimensions. Immediately following introduction of the DLPC stock solution, the $\mathrm{OH}$ SS-S intensity drops by almost a factor of 10 in-plane and to almost zero out-of-plane. Spectra acquired under SPS conditions show that the $\mathrm{OH}$ SS-A also experiences a precipitous reduction in intensity immediately after the monolayer begins to form. (Data not shown). Water molecules contributing to the observed sum frequency intensity at low DLPC surface coverages can be involved in solvation of the phospholipid head-groups. The observed reduction in intensity is likely due to a combination of factors including both a reduction of the number of contributing $\mathrm{OH}$ oscillators 
(based on the correlation between the in-plane and out-of-plane intensity fluctuations) and the possible re-orientation of solvating and non-solvating interfacial water molecules. Adsorption of the DLPC monomers will alter the interfacial potential [26] which, in turn may influence the orientation of water molecules not necessarily involved in solvating the head-group. The neat water- $\mathrm{CCl}_{4}$ interface starts out with a calculated surface potential of $\sim 150 \mathrm{mV}$ [36].

Between $\sim 15 \mathrm{~min}$ and $2 \mathrm{~h} \mathrm{OH} \mathrm{SS-S} \mathrm{intensity}$ in both in-plane and out-of-plane dimensions oscillates in a complicated but systematic and reproducible fashion. Based on intensity fluctuations arising from different functional groups belonging to the adsorbed DLPC monomers we believe these changes in $\mathrm{OH}$ SS-S intensity arise from solvent response to two-dimensional phase transitions in the DLPC monolayer [24,25]. Neutron scattering experiments carried out on phosphocholine monolayers adsorbed to the air-water interface find that the extent of head-group and backbone hydration sensitively depends on surface concentration [37]. Expanded monolayers are

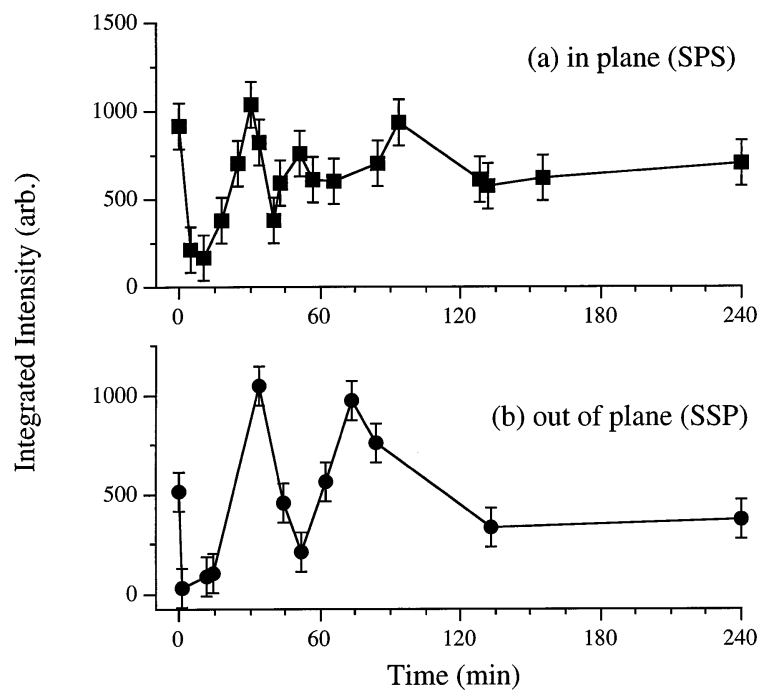

Fig. 5. Time-dependent intensity in the OH SS-S band (a) in the plane of the interface (SPS) and (b) out of the plane of the interface (SSP). Intensities have been corrected for power and for differences in Fresnel coefficients. Error bars reflect a combination of uncertainties in the spectral fitting and experimental reproducibility. solvated by the aqueous phase all the way up to the carboxyl groups or to a depth of $\sim 8 \AA$. The three carbon glycero backbone is spread in an extended, 'butterfly' orientation. At higher surface concentrations, the glycero backbone undergoes a drastic conformational change to become more compact. This re-structuring 'lifts' the carboxyl groups from the aqueous phase and leads to a hydration depth of only $\sim 4 \AA$. Such changes in adsorbed DLPC conformation will inevitably affect the surrounding structure of the solvating water molecules as well as those water molecules outside of the first solvation sphere.

Although earlier work [26] demonstrated how vibrational motion in the $\mathrm{CH}$ stretching region could constructively or destructively interfere with VSF intensity in the water stretching region, we do not believe that such interference is responsible for the observed intensity fluctuations in the $\mathrm{OH}$ SS-S band. Interaction between $\mathrm{CH}$ and $\mathrm{OH}$ vibrational modes arises when a simple, soluble, charged surfactant (i.e. sodium dodecyl sulfate or dodecyl ammonium chloride) adsorbs to an interface. Together with their counterions, these singly-charged surfactants set up a strong potential gradient normal to the interface which orients water molecules to a depth of approximately the Debye screening length [26]. The extent of spectral interference is directly related to the number of contributing oscillators; a stronger gradient over a longer distance necessarily leads to greater interaction between the $\mathrm{CH}$ and $\mathrm{OH}$ vibrational modes. We observe no evidence of interference between the $\mathrm{CH}$ and $\mathrm{OH}$ stretching regions of the spectra, consistent with a much weaker or non-existent potential gradient normal to the interface [26].

Phosphocholine head-groups carry a zwitterionic charge and spectral data show that they lie parallel to the interface at all surface concentrations in agreement with calculations $[38,39]$. Consequently, the DLPC monolayer shares many similarities with monolayers formed from a mixture of simple anionic and cationic surfactants. In VSFS studies of these mixed surfactant systems, spectra show no indication of interference between the very weak $\mathrm{OH}$ response and the $\mathrm{CH}$ modes of the surfactant chains [26]. Finally, if 
interference effects were present in our data, we would expect to see differences between the SPS and SSP intensity data based on the different dimensions these polarization conditions sample. Fig. 3 shows that the oscillations in the OH SS-S intensities mirror each other in the in-plane and out-of-plane dimensions, implying that interference between the $\mathrm{CH}$ and $\mathrm{OH}$ vibrational modes is not contributing to the observed intensity fluctuations.

\subsection{Temporal dependence of $\mathrm{OH} S \mathrm{~S}-\mathrm{S}$ frequency}

In addition to exhibiting intensity fluctuations, the $\mathrm{OH}$ SS-S also shifts in frequency as DLPC monomers adsorb to the interface (Fig. 5). The frequency of the $\mathrm{OH}$ SS-S band provides a very sensitive measure of the extent of hydrogen bonding [27,32,40]. Infrared [31] and VSF [27] studies have shown that stronger hydrogen bonding leads to a lower OH SS-S frequency. Stronger hydrogen bonding results in a lengthening of the covalent $\mathrm{OH}$ bonds and a corresponding red shift in the $\mathrm{OH}$ SS-S frequency. Conversely, aqueous systems with weak hydrogen bonding typically show the $\mathrm{OH}$ SS-S at higher frequencies.[31]

Before the addition of DLPC, the in-plane $\mathrm{OH}$ SS-S appears at $3250 \mathrm{~cm}^{-1}$ indicating weak, inplane hydrogen bonding. (Fig. 6a) Immediately after the monolayer begins to form, however, this band experiences a sharp, $\sim 100 \mathrm{~cm}^{-1}$ shift to lower energy. This result suggests that adsorption of DLPC monomers to the aqueous- $\mathrm{CCl}_{4}$ interface strengthens hydrogen bonding between water molecules aligned parallel to the interface. As the DLPC monolayer continues to form, the in-plane $\mathrm{OH}$ SS-S band remains close to $3140 \mathrm{~cm}^{-1}$, the frequency attained within the first $10 \mathrm{~min}$ of monolayer formation.

The out-of-plane OH SS-S frequency abruptly shifts $30 \mathrm{~cm}^{-1}$ to higher energy following addition of the DLPC stock solution. (Fig. 6b) This blue shift indicates that adsorption of the first DLPC monomers disrupts the out-of-plane hydrogen bonding. However, unlike the in-plane $\mathrm{OH}$ SS-S which remains at its new frequency, the out-of-plane $\mathrm{OH}$ SS-S gradually returns to its original position $\left(3180 \mathrm{~cm}^{-1}\right)$ approximately $1 \mathrm{~h}$

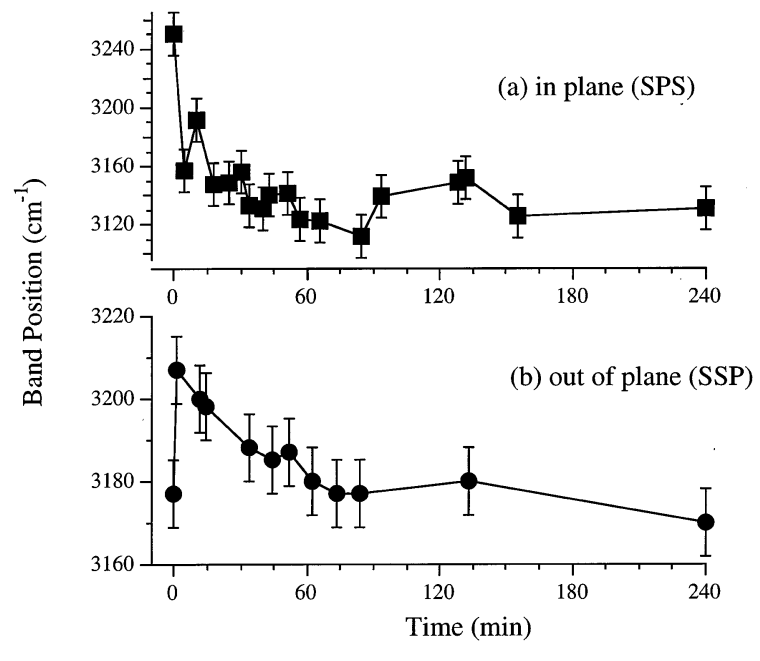

Fig. 6. Time-dependent center position of the OH SS-S band (a) in the plane of the interface (SPS) and (b) out of the plane of the interface (SSP). Band positions have been calibrated against a monochrometer and uncertainties reflect a combination of $\omega_{\text {ir }}$ bandwidth $\left(18 \mathrm{~cm}^{-1}\right)$ and reproducibility of spectral fitting.

after the monolayer begins to form. Thus, although adsorption of DLPC monomers appears to reduce the degree of hydrogen bonding normal to the interface, the hydrogen bonding network gradually strengthens as monolayer formation proceeds. Based on the center position of the out-of-plane OH SS-S feature, the extent of this out-of-plane hydrogen bonding appears to be quite similar for both the neat interface and the interface with the equilibrated, tightly packed DLPC monolayer.

From the intensity and the frequency data, we conclude that the out-of-plane water structure is quite similar before and after monolayer formation. The intensity and the frequency of the outof-plane plane OH SS-S are approximately the same at $t=0$ (pre-monolayer) and $t=\infty$ (equilibrated monolayer). The formation process itself, however, induces large fluctuations in the out-ofplane environment as interfacial water molecules respond to re-orientations of adsorbed DLPC monomers induced by the ever increasing surface concentration.

Intensity data show that the in-plane environment experiences large fluctuations similar to 
those experienced in the out-of-plane dimension. However, water molecules aligned parallel to the interface experience strong, irreversible strengthening in their hydrogen bonding immediately after the monolayer begins to form. Taking these observations one step further, we speculate that the orientation of the DLPC head-groups bear responsibility for the observed effects. Head-groups arranged parallel to the interface will have domains of large electrostatic potential between them. These potential gradients can align solvating water molecules in a parallel orientation and stabilize in-plane water hydrogen bonding. Similar ideas have been invoked to explain pH-dependent re-orientations of fatty acid head-groups at the air-water interface [41].

We note that molecular dynamics simulations have extensively examined the structure and dynamics of water molecules between phosphocholine bilayers [42-45] These studies find that the first 1-2 layers of water molecules align with their transition dipoles normal to the interfacial plane, contrary to our VSF studies which suggest a significant in-plane alignment of water molecules. However, several important differences exist between the systems studied in this work and the systems modeled in the simulations. In the simulations the presence of a second head-group 'sheet' located only Angstroms away from the first introduces strong effects into the surrounding solvent structure. For the monolayer studies presented above, no such perturbative source is present to influence the water molecules solvating the head-groups. Also, the simulations begin with phosphocholine head-groups packed more closely together (47.4 $\AA^{2} /$ molecule) than the tightly packed monolayers formed in the experiments described above (55 $\AA^{2}$ /molecule). This difference in monomer areas is enough to allow approximately one water molecule per DLPC monomer to intercalate between the head-groups of the adsorbed species. Assuming the head-groups to lie parallel to the interface, any intercalated water molecules will experience very strong asymmetric forces in the in-plane dimension. This environment would necessarily lead to a strong non-linear response consistent with experimental data.

\section{Conclusion}

The experiments described in this work detail how vibrational sum frequency spectroscopy can monitor the real time dynamics of interfacial solvent structure during the formation of a phospholipid monolayer at a liquid-liquid interface. VSFS spectra represent snapshots of the vibrational structure of molecules in the interfacial region at different stages of the monolayer formation process. Intensity changes and spectral shifts of vibrational bands allow observation of how solvent environment evolves as the monolayer slowly forms an equilibrated, condensed, two-dimensional structure.

Dynamic surface tension measurements suggest that monolayer formation proceeds in a well defined, continuous fashion with the surface tension smoothly changing from that of the neat interface to that of the equilibrated, tightly packed monolayer without any discontinuities. Data from VSFS spectra tell a different story. During the first several hours of monolayer formation - a process which takes $\sim 6 \mathrm{~h}$ for a 4.5 $\mu \mathrm{M}$ DLPC aqueous phase at room temperaturespectra of the $\mathrm{OH}$ stretching region show pronounced intensity oscillations both in the plane of the interface and normal to the interface. Initial adsorption of phosphocholine monomers to the aqueous $-\mathrm{CCl}_{4}$ interface appears to disrupt the hydrogen bonding structure of the interfacial water molecules. The oscillations in $\mathrm{OH}$ SS-S intensity which occur during the next $2 \mathrm{~h}$ may correlate with two-dimensional phase transitions within the monolayer and the resulting re-orientation of the adsorbed monomers.

Frequency shifts in the OH SS-S band also shed light into how the solvent environment changes as the monolayer forms. A dramatic red shift in the $\mathrm{OH}$ SS-S vibrational band assigned to those water molecules having their symmetry axes parallel to the interface provides compelling evidence that the zwitterionic DLPC head-groups lie parallel to the interface setting up microscopic regions of strong potential gradients. These gradients may serve to align and stabilize water molecules positioned between the head-groups. For water molecules oriented normal to the interface, the 
initial stages of monolayer formation abruptly reduces the extent of hydrogen bonding leading to a blue shift in the out-of-plane OH SS-S frequency. Eventually, the out-of-plane hydrogen bonding network re-establishes itself and the outof-plane $\mathrm{OH}$ SS-S returns to its pre-monolayer frequency.

These experiments demonstrate the feasibility of using interfacially specific, non-linear optical spectroscopic methods to probe the dynamics of interfacial processes. Furthermore, the results show that molecular re-orientation which accompanies adsorption of complex surfactants to interfaces can be quite complicated. We are currently examining in greater detail the spectroscopic changes which accompany phospholipid monolayer formation at a water- $\mathrm{CCl}_{4}$ interface. Preliminary results indicate that the slower kinetics leads to dampened spectral intensity fluctuations.

\section{Acknowledgements}

The authors deeply appreciate the preliminary work of Lawrence Scatena examining the free $\mathrm{OH}$ stretch in VSF spectra of the neat aqueous $-\mathrm{CCl}_{4}$ interface. Support from the National Science Foundation (CHE-9725751) is gratefully acknowledged.

\section{References}

[1] J. Israelachvili, Intermolecular and Surface Forces, Academic Press Inc, San Diego, 1995.

[2] D. Myers, Surfaces, Interfaces and Colloids: Principles and Applications, vol. 1, VCH Publishers Inc, New York, 1991.

[3] C.A. Miller, Interfacial Phenomena, Marcel Dekker Inc, New York, 1985.

[4] P.C. Hiemenz, R. Rajagopalan, Principles of Colloid and Surface Chemistry, Marcel Dekker Inc, New York, 1997.

[5] J. Li, V.B. Fainerman, R. Miller, Langmuir 12 (1996) 5138.

[6] J.T. Davies, E.K. Rideal, Interfacial Phenomena, Academic Press Inc, New York, 1963.

[7] T. Rasing, T. Stehlin, Y.R. Shen, M.W. Kim, J.P. Valint, J. Chem. Phys. 89 (1988) 3386-3387.

[8] C.D. Bain, J. Chem. Soc. Faraday Trans. 91 (1995) 1281-1296.
[9] R.M. Corn, D.A. Higgins, Chem. Rev. 94 (1994) $107-$ 125.

[10] K.B. Eisenthal, Chem. Rev. 96 (1996) 1343-1360.

[11] G.L. Richmond, Anal. Chem. 69 (1997) 536A-543A.

[12] Y.R. Shen, Solid State Comm. 102 (1997) 221-229.

[13] D. Chapman, in: G. Gregoriadis (Ed.), Liposome Technology, 1, CRC Press, Boca Raton, 1983, pp. 1-18.

[14] R.C. MacDonald, S.A. Simon, Proc. Natl. Acad. Sci. USA 84 (1987) 4089-4093.

[15] B.A. Denizot, P.C. Tchoreloff, J.E. Proust, F. Puisieux, A. Lindenbaum, M. Dehan, J. Colloid Interface Sci. 143 (1991) 120-126.

[16] K. Nag, J. Perez-Gil, A. Cruz, N.H. Rich, K.M.W. Keough, Biophys. J. 71 (1996) 1356-1363.

[17] R. Qiu, R.C. MacDonald, Biochim. Biophys. Acta 1191 (1994) 343-353.

[18] R.A. Walker, G.L. Richmond, Langmuir (submitted for publication).

[19] J.C. Conboy, M.C. Messmer, G.L. Richmond, J. Phys. Chem. 100 (1996).

[20] B. Dick, A. Gierulski, G. Marowsky, G.A. Reider, Appl. Phys. B 38 (1985) 107-116.

[21] N. Bloembergen, P.S. Pershan, Phys. Rev. 128 (1962) 606-622.

[22] D.E. Gragson, D.S. Alavi, Opt. Lett. 20 (1995) 19911995.

[23] R.A. Walker, J.C. Conboy, G.L. Richmond, Langmuir 13 (1997) 3070-3073.

[24] M. Thoma, H. Mohwld, J. Colloid Interface Sci. 162 (1994) 340.

[25] J. Mingins, J.A. Taylor, B.A. Pethica, C.M. Jackson, B.Y.T. Yue, J. Chem. Soc. Faraday Trans. 78 (1982) 323.

[26] D.E. Gragson, B.M. McCarty, G.L. Richmond, J. Am. Chem. Soc. 119 (1997) 6144-6152.

[27] D.E. Gragson, G.L. Richmond, J. Chem. Phys. 107 (1997) 9687-9690.

[28] Q. Du, E. Freysz, Y.R. Shen, Phys. Rev. Lett. 72 (1994) 238-241.

[29] Q. Du, E. Freysz, Y.R. Shen, Science 264 (1994) 826-828.

[30] Q. Du, R. Superfine, E. Freysz, Phys. Rev. Lett. 70 (1993) 2313-2316.

[31] J.R. Scherer, in: R.J.H. Clark, R.E. Hester (Eds.), Advances in Infrared and Raman Spectroscopy, vol. 5, Heyden, Philadelphia, 1978, pp. 149-216.

[32] G.A. Jeffrey, An Introduction to Hydrogen Bonding, Oxford University Press, New York, 1997.

[33] P.A. Gignere, J. Raman Spec. 15 (1984) 384.

[34] D.E. Gragson, G.L. Richmond, J. Phys. Chem. B. 102 (1998) 3847-3861.

[35] L.F. Scatena, G.L. Richmond (in preparation).

[36] T.M. Chang, L.X. Dang, J. Chem. Phys. 104 (1996) $6772-6783$

[37] T.M. Bayerl, R.K. Thomas, J. Penfold, A. Rennie, E. Sackmann, Biophys. J. 57 (1990) 1095-1098.

[38] D. Stigter, K.A. Dill, Langmuir 4 (1988) 200-209.

[39] K.A. Dill, D. Stigter, Biochemistry 27 (1988) 3446-3453. 
[40] G.E. Walrafen, in: F. Franks (Ed.), Water: A Comprehensive Treatise, vol. 1, Plenum, New York, 1972, pp. $151-254$.

[41] P.B. Miranda, Q. Du, Y.R. Shen, Chem. Phys. Lett. 286 (1998) $1-8$.

[42] U. Essmann, L. Perera, M.L. Berkowitz, Langmuir 11 (1995) 4519-4531.
[43] K. Raghavan, M. Rami-Reddy, M.L. Berkowitz, Langmuir 8 (1992) 233-240.

[44] L. Perera, U. Essman, M.L. Berkowitz, Langmuir 12 (1996) 2625-2629.

[45] S.W. Chiu, M. Clark, V. Balaji, S. Subramaniam, H.S. Scott, E. Jakobsson, Biophys. J. 69 (1995). 